\title{
Study of nuclear cataract progression using the National Eye Institute Scheimpflug system
}

\author{
Manuel B Datiles III, Benjamin V Magno, Valeria Freidlin
}

\begin{abstract}
Aims-A study was conducted to determine the capability of the recently developed National Eye Institute (NEI) Scheimpflug cataract imaging system in detecting changes in the nuclear region of the lens over a 1 year period.

Methods-Twenty five eyes with pure nuclear cataracts with mean nuclear densities $\leqslant 0.30$ optical density units (ODU) as well as 30 normal control eyes were each examined at baseline and 12 months later. Computerised densitometry using the NEI Scheimpflug cataract imaging system was performed. Clinical grading of the lenses was also done using the Lens Opacities Classification System II (LOCS II). For densitometry, a change of plus or minus 0.023 ODU (the $99 \%$ range) in mean density in the nuclear area was considered a progression or regression at 1 year.
\end{abstract}

Results-Using the Scheimpflug densitometry, 14 of the 25 cataractous eyes showed significant progression at 1 year. In the normal control group, only three of the 30 eyes showed significant progression. In contrast, using the LOCS II clinical grading, only two of the 25 cataractous eyes showed a one step increase, two of the 30 controls progressed at 1 year, and none regressed. There was no significant difference in visual acuity.

Conclusion-This study suggests the value of the NEI Scheimpflug cataract imaging system in detecting nuclear change within 1 year. However, clinically significant changes may require longer follow up periods. These data will be useful in planning future longitudinal studies of nuclear cataracts, such as for clinical trials of anticataract drugs.

(Br f Ophthalmol 1995; 79: 527-534)

National Eye Institute,

Health, Bethesda, USA

Cataract and Corneal

Disease Section,

Ophthalmic Genetics

and Clinical Services

Branch

M B Datiles III

B V Magno

Division of Biometry and Epidemiology

V Freidlin

Correspondence to:

Manuel B Datiles III, MD, NEI-NIH, 10 Center DR MSC 1860, Building 10, Room 10N226, Bethesda, MD 20892-1860, USA.

Accepted for publication 10 January 1995 (Topcon, Oxford, and Nidek systems) ${ }^{5-17}$ and slit-lamp photography, ${ }^{18} 2022$ but the question remains - how sensitive are these systems in detecting actual changes in nuclear cataract progression? In addition, there is a paucity of published data on longitudinal studies of cataracts, as well as related data needed in designing future clinical trials of anti-cataract drugs, such as relative rates of progression of various types of cataracts and suitability of various methods to monitor those progressions.

Scheimpflug cataract imaging (an objective cataract grading method, which uses a modified slit beam), has been used to study the ocular lens for the past two decades, and its capabilities, as well as limitations, has been well documented and explored. ${ }^{2-15}$ It has been found to be best for studying the nuclear region of the lens, and to be less reproducible in studies of the anterior cortex, posterior cortex, and posterior subcapsular area. ${ }^{7-15} \mathrm{We}$ have therefore confined this present study using the Zeiss Scheimpflug camera (Carl Zeiss Inc, Oberkochen, Germany) on the nuclear region of the lenses of normal volunteers as well as cataractous patients.

We have developed the National Eye Institute (NEI) Scheimpflug cataract imaging system $^{16} 17$ which was used to analyse all images in this study. A recent publication reported on the reproducibility of this method on the lens nucleus using the $99 \%$ range, ${ }^{17}$ which is defined as the interval within which $99 \%$ of the values of repeat measurements may be expected to lie. The results of that study showed that for lenses with nuclear densities less than 0.30 optical density units (ODU), the $99 \%$ range of plus or minus 0.023 ODU was obtained. For lenses with nuclear densities over 0.30 ODU, the $99 \%$ range was plus or minus 0.14 ODU times the first measurement. The NEI Scheimpflug cataract imaging system was developed to make longitudinal studies more practical to conduct, and more reliable, and these will be dealt with further in the discussion.

For clinical grading in this study, we used the Lens Opacities Classification II (LOCS II). ${ }^{18} \mathrm{We}$ have previously reported on our experience with using the LOCS II (a subjective grading method) for clinically grading cataracts and studying cataract progression ${ }^{19}$ and we have obtained good reproducibility with this method. Most other lens grading systems use similar scales for grading nuclear cataracts photographically and clinically (Oxford, ${ }^{7}$ Wilmer, ${ }^{20}$ Wisconsin ${ }^{21}$ ).

We therefore conducted a 1 year longitudinal study of age-related nuclear cataracts, using the NEI Scheimpflug cataract imaging system, to determine its capability to detect changes in nuclear density over the 1 year period, compared with the LOCS II and visual acuity. For this purpose, we sought a group of early to moderately advanced 


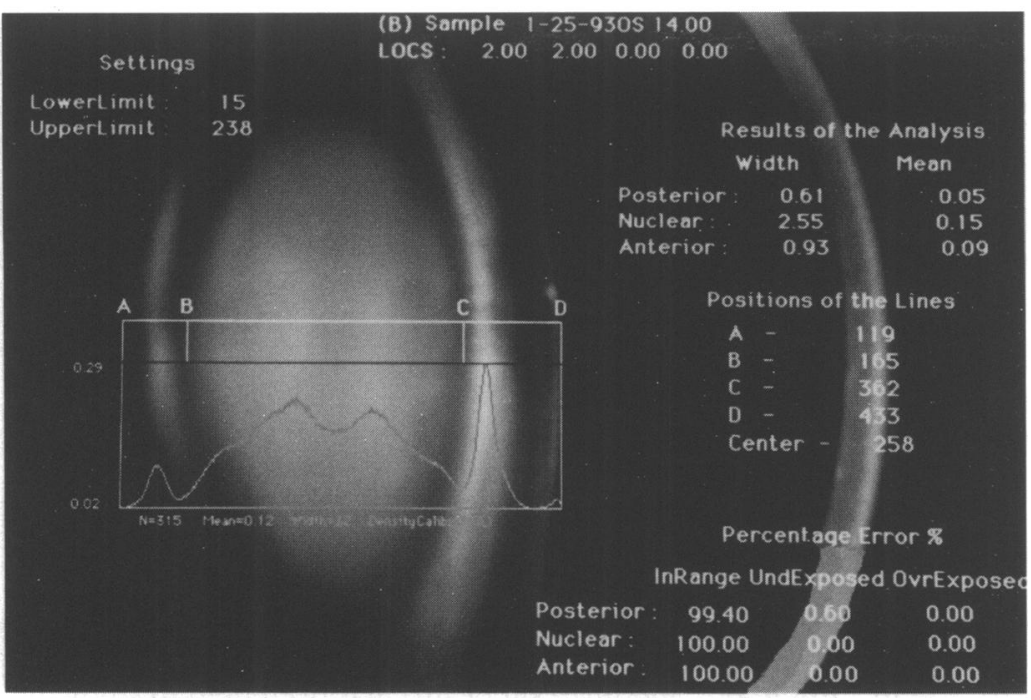

Figure 1 Example of a Scheimpflug image of a LOCS II grade 2 nuclear cataract with densitometry readings (in optical density units, ODU) using the NEI Scheimpflug cataract imaging system.

nuclear cataracts, to give allowances for future progression as we followed the cataracts over time.

\section{Materials and methods}

We studied 25 eyes with pure nuclear cataracts with nuclear densities equal to or less than 0.30 ODU on the first visit, and 30 eyes with clear lenses as controls. The age of cataract patients ranged from 48 to 85 (mean 68) years, and for normals, 22-74 (mean 50) years. Fourteen of the 30 controls were aged 50 and above. Owing to the scarcity of normal clear lenses in the older age group, we did not deem it essential to use age-matched controls, but emphasised lens status as the critical criterion. These patients were participants in a National Eye Institute intramural review board approved study being conducted at the eye clinic of the National Eye Institute and gave full informed consent. We strictly adhered to the tenets of the Helsinki Declaration. Only the left eyes were used for this study, unless the left eye was post cataract surgery, had a nuclear cataract more than 0.30 ODU, or had other ocular localised disorders such as corneal, tear, lid, or iris disorders. In these instances, the right eye was used (total right eyes nine from nuclear cataract group and four from normal control group). The patients and controls underwent a complete eye examination with maximum dilatation of the pupils. Best corrected visual acuity was obtained using the ETDRS chart ${ }^{23}$ before dilatation. The clinical grading of the cataracts was performed in a masked fashion by one of the authors (MBD) at the Haag Streit slit-lamp, using the LOCS II. ${ }^{18}$ Scheimpflug photography was performed with the Zeiss Scheimpflug slit-lamp video camera and digitised using the NEI Scheimpflug cataract imaging software. ${ }^{16}$

Images obtained with the Zeiss Scheimpflug video camera were stored in an optical disk and analysed using a Macintosh Quadra 700 computer (Apple Computers Inc, Cupertino, CA, USA). Each lens image was then automatically subdivided by the NEI Scheimpflug cataract imaging system into anterior cortical, nuclear, and posterior cortical subcapsular anatomical regions ${ }^{1617}$ (Fig 1). Mean density measurements were obtained by a rectangular region of interest ( 32 pixels in height) along the axial centre of the nucleus. All data were expressed in optical density units using a specially developed calibration system, ${ }^{16}$ and each image was checked thoroughly for proper exposure and alignment at the time of photography before storage and analysis.

Table 1 Normal control data, showing LOCS II clinical grading of nuclear opalescence, and Scheimpflug densitometry readings in the nuclear areas for initial and 12 month visits and visual acuity

\begin{tabular}{|c|c|c|c|c|c|c|c|c|c|c|c|c|}
\hline $\begin{array}{l}\text { Case } \\
\text { No }\end{array}$ & $\begin{array}{l}\text { Age } \\
\text { (years) }\end{array}$ & Sex & $\begin{array}{l}\text { Date } \\
\text { visit } 1\end{array}$ & Eye & $\begin{array}{l}\text { LOCS II } \\
\text { NO }\end{array}$ & $\begin{array}{l}\text { Scheimpflug } \\
\text { nuclear density }\end{array}$ & $\begin{array}{l}\text { Visual acuity } \\
\text { (No of letters) }\end{array}$ & $\begin{array}{l}\text { Date } \\
\text { Visit } 2\end{array}$ & Eye & $\begin{array}{l}\text { LOCS II } \\
\text { NO }\end{array}$ & $\begin{array}{l}\text { Scheimpflug } \\
\text { nuclear density }\end{array}$ & $\begin{array}{l}\text { Visual acuity } \\
\text { (No of letters) }\end{array}$ \\
\hline $\begin{array}{r}1 \\
2 \\
3 \\
4 \\
5 \\
6 \\
7 \\
8 \\
9 \\
10 \\
11 \\
12 \\
13 \\
14 \\
15 \\
16 \\
17 \\
18 \\
19 \\
20 \\
21 \\
22 \\
23 \\
24 \\
25 \\
26 \\
27 \\
28 \\
29 \\
30\end{array}$ & $\begin{array}{l}43 \cdot 0 \\
64 \cdot 2 \\
42 \cdot 3 \\
61 \cdot 2 \\
59 \cdot 4 \\
63 \cdot 6 \\
45 \cdot 5 \\
56 \cdot 2 \\
61 \cdot 5 \\
22 \cdot 1 \\
47 \cdot 6 \\
44 \cdot 1 \\
58 \cdot 3 \\
56 \cdot 5 \\
68 \cdot 1 \\
51 \cdot 5 \\
31 \cdot 3 \\
67 \cdot 7 \\
36 \cdot 1 \\
38 \cdot 7 \\
43 \cdot 4 \\
23 \cdot 1 \\
55 \cdot 0 \\
62 \cdot 8 \\
56 \cdot 5 \\
59 \cdot 8 \\
22 \cdot 5 \\
37 \cdot 2 \\
45 \cdot 1 \\
73 \cdot 5\end{array}$ & $\begin{array}{l}\mathbf{F} \\
\mathbf{M} \\
\mathbf{F} \\
\mathbf{M} \\
\mathbf{F} \\
\mathbf{M} \\
\mathbf{M} \\
\mathbf{F} \\
\mathbf{M} \\
\mathbf{M} \\
\mathbf{F} \\
\mathbf{M} \\
\mathbf{F} \\
\mathbf{F} \\
\mathbf{F} \\
\mathbf{F} \\
\mathbf{F} \\
\mathbf{F} \\
\mathbf{F} \\
\mathbf{F} \\
\mathbf{M} \\
\mathbf{F} \\
\mathbf{F} \\
\mathbf{M} \\
\mathbf{F} \\
\mathbf{F} \\
\mathbf{M} \\
\mathbf{F} \\
\mathbf{F} \\
\mathbf{M}\end{array}$ & $\begin{array}{l}8 / 2 / 91 \\
7 / 8 / 91 \\
8 / 26 / 91 \\
9 / 25 / 91 \\
9 / 23 / 91 \\
10 / 21 / 91 \\
6 / 17 / 91 \\
6 / 19 / 91 \\
11 / 6 / 91 \\
7 / 24 / 89 \\
6 / 10 / 91 \\
5 / 6 / 91 \\
11 / 4 / 91 \\
5 / 21 / 91 \\
4 / 1 / 91 \\
3 / 11 / 91 \\
6 / 19 / 91 \\
4 / 1 / 91 \\
7 / 8 / 91 \\
7 / 12 / 91 \\
3 / 1 / 91 \\
7 / 19 / 91 \\
6 / 17 / 91 \\
8 / 12 / 91 \\
6 / 24 / 91 \\
9 / 9 / 91 \\
12 / 9 / 91 \\
10 / 21 / 91 \\
9 / 25 / 91 \\
6 / 10 / 91\end{array}$ & $\begin{array}{l}\text { L } \\
\text { L } \\
\text { L } \\
\text { L } \\
\text { R } \\
\text { L } \\
\text { L } \\
\text { L } \\
\text { L } \\
\text { L } \\
\text { L } \\
R \\
\text { L } \\
\text { L } \\
\text { L } \\
\text { L } \\
\text { L } \\
R \\
R \\
\text { L } \\
\text { L } \\
\text { L } \\
\text { L } \\
\text { L } \\
\text { L } \\
\text { L } \\
\text { L } \\
\text { L } \\
\text { L } \\
\text { L }\end{array}$ & $\begin{array}{l}0 \\
0 \\
0 \\
0 \\
0 \\
0 \\
0 \\
0 \\
0 \\
0 \\
0 \\
0 \\
0 \\
0 \\
0 \\
0 \\
0 \\
0 \\
0 \\
0 \\
0 \\
0 \\
0 \\
0 \\
0 \\
0 \\
0 \\
0 \\
0 \\
0\end{array}$ & $\begin{array}{l}0.03 \\
0.06 \\
0.03 \\
0.06 \\
0.05 \\
0.04 \\
0.05 \\
0.04 \\
0.03 \\
0.03 \\
0.03 \\
0.06 \\
0.04 \\
0.06 \\
0.08 \\
0.04 \\
0.03 \\
0.07 \\
0.04 \\
0.04 \\
0.05 \\
0.03 \\
0.06 \\
0.06 \\
0.04 \\
0.06 \\
0.03 \\
0.04 \\
0.01 \\
0.06\end{array}$ & $\begin{array}{l}55 \\
55 \\
54 \\
65 \\
54 \\
57 \\
45 \\
55 \\
55 \\
60 \\
59 \\
40 \\
57 \\
59 \\
55 \\
60 \\
59 \\
60 \\
55 \\
50 \\
46 \\
53 \\
44 \\
58 \\
60 \\
60 \\
65 \\
58 \\
54 \\
50\end{array}$ & $\begin{array}{l}8 / 14 / 92 \\
7 / 13 / 92 \\
8 / 17 / 92 \\
9 / 30 / 92 \\
9 / 28 / 92 \\
10 / 26 / 92 \\
6 / 22 / 92 \\
6 / 10 / 92 \\
11 / 16 / 92 \\
6 / 10 / 90 \\
6 / 15 / 92 \\
5 / 18 / 92 \\
11 / 18 / 92 \\
5 / 13 / 92 \\
5 / 18 / 92 \\
3 / 16 / 92 \\
7 / 29 / 92 \\
4 / 13 / 92 \\
7 / 20 / 92 \\
7 / 29 / 92 \\
3 / 2 / 92 \\
8 / 19 / 92 \\
5 / 20 / 92 \\
8 / 10 / 92 \\
5 / 22 / 92 \\
9 / 30 / 92 \\
12 / 9 / 92 \\
9 / 21 / 92 \\
9 / 30 / 92 \\
6 / 29 / 92\end{array}$ & $\begin{array}{l}\text { L } \\
\text { L } \\
\text { L } \\
\text { L } \\
\text { R } \\
\text { L } \\
\text { L } \\
\text { L } \\
\text { L } \\
\text { L } \\
\text { L } \\
\text { R } \\
\text { L } \\
\text { L } \\
\text { L } \\
\text { L } \\
\text { L } \\
\text { R } \\
R \\
\text { L } \\
\text { L } \\
\text { L } \\
\text { L } \\
\text { L } \\
\text { L } \\
\text { L } \\
\text { L } \\
\text { L } \\
\text { L } \\
\text { L }\end{array}$ & $\begin{array}{l}0 \\
0 \\
0 \\
0 \\
0 \\
0 \\
0 \\
0 \\
0 \\
0 \\
0 \\
0 \\
0 \\
0 \\
0 \\
0 \\
0 \\
0 \\
0 \\
0 \\
0 \\
0 \\
0 \\
1 \\
0 \\
0 \\
0 \\
0 \\
0 \\
1\end{array}$ & $\begin{array}{l}0.03 \\
0.07 \\
0.03 \\
0.05 \\
0.04 \\
0.03 \\
0.04 \\
0.06 \\
0.03 \\
0.02 \\
0.03 \\
0.05 \\
0.02 \\
0.05 \\
0.11^{\star} \\
0.04 \\
0.03 \\
0.08 \\
0.03 \\
0.02 \\
0.04 \\
0.01 \\
0.09 \star \\
0.08 \\
0.03 \\
0.05 \\
0.02 \\
0.03 \\
0.02 \\
0.09 \star\end{array}$ & $\begin{array}{l}57 \\
55 \\
60 \\
60 \\
48 \\
55 \\
46 \\
58 \\
56 \\
62 \\
60 \\
44 \\
53 \\
60 \\
55 \\
65 \\
52 \\
60 \\
60 \\
54 \\
56 \\
59 \\
46 \\
54 \\
55 \\
53 \\
65 \\
60 \\
55 \\
50\end{array}$ \\
\hline
\end{tabular}

*Significant increase in density. LOCS II NO=LOCS II clinical grading of nuclear opalescence; visual acuity (no of letters): best corrected visual acuity using the number of letters read using the ETDRS chart ( 5 letters per line) eq: $20 / 20=55,20 / 25=50,20 / 32=45,20 / 40=40,20 / 50=35$ letters, etc. A difference of 10 letters is considered a significant change. 


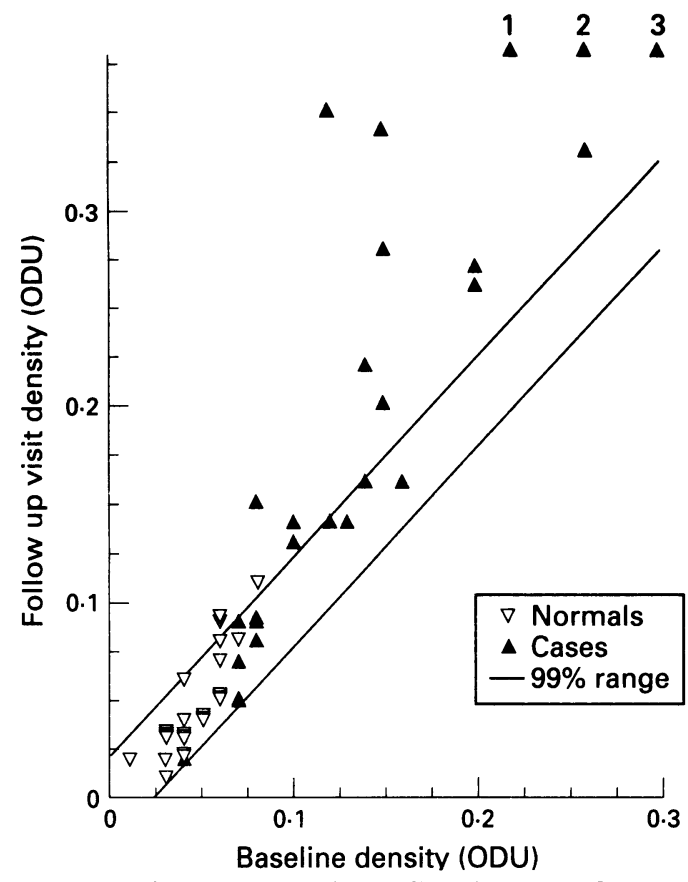

Figure 2 Graph showing the baseline visit versus the 12 month follow up visit for both normal $(\nabla)$ and nuclear cataract eyes $(\boldsymbol{\Delta})$. The diagonal lines delineate the $99 \%$ range of the system. Points 1, 2, and 3 correspond to cataract case numbers 1,14 , and 6 , representing eyes which started below $0 \cdot 30$ ODU at the first visit and progressed beyond 0.38 ODU limit of the graph and were just lined up on the margin.

The patients and controls were examined and photographed in the same way 12 (SD 2) months later.

The Scheimpflug data were analysed using the $99 \%$ range of plus or minus $0 \cdot 23$ ODU $^{17}$ for nuclear densities below 0.30 ODU. The nuclear cataract was considered to have progressed (or regressed) if a change in the density measurement from baseline was beyond 0.023 ODU. If the change in the density measurement from baseline was within plus or minus $0.023 \mathrm{ODU}$, then this difference was attributed to the measurement error and no change in the cataract status was indicated.

We plotted the 12 month measurements against baseline densities. On this plot, each person is represented by a point with coordinates corresponding to the paired measurements. The $99 \%$ range of plus or minus 0.023 ODU can be represented by the region between two parallel diagonal lines which contains all the points for which the difference between two coordinates is within 0.023 ODU.

For the clinical LOCS II grading system, a clinical decision is made during the examination of the patient, and because it cannot be verified at a later date, we have developed a rule wherein a change of one or more steps in the 12 month visit would need to be confirmed by a third visit. ${ }^{19}$ Therefore, patients with changes at 12 months from baseline were examined again for a third visit 18-24 months from baseline for the confirmatory LOCS II grading.

\section{Results}

Table 1 shows the data obtained from the normal control group, which include the dates of visit, the LOCS II clinical grading, as well as the corresponding Scheimpflug nuclear densities and number of letters on the ETDRS chart read at each visit. Table 2 similarly shows the data obtained from the nuclear cataract group.

Figure 2 summarises the results. The nuclear density at baseline for each eye was plotted against the nuclear density at the 12 month follow up visit. Note in this figure that the normal controls (meaning clear lenses with corresponding LOCS II clinical grade of 0 ) are distributed mainly around the lower density values, whereas the nuclear cataract group were distributed mainly in the higher densities. The area within the diagonal lines represents the $99 \%$ range obtained from the reproducibility study ${ }^{17}$ which contains all points for which the difference between the measurements at baseline and the 12 month visit is within 0.023 ODU. Therefore, points above or below these lines can be considered progression or regression, respectively.

As shown in Figure 2 and Table 2, 14 of the 25 cataractous eyes significantly increased in

Table 2 Nuclear cataract data, as in Table 1

\begin{tabular}{|c|c|c|c|c|c|c|c|c|c|c|c|c|}
\hline $\begin{array}{l}\text { Case } \\
\text { No }\end{array}$ & $\begin{array}{l}\text { Age } \\
\text { (years) }\end{array}$ & Sex & $\begin{array}{l}\text { Date } \\
\text { visit } 1\end{array}$ & Eye & $\begin{array}{l}\text { LOCS II } \\
\text { NO }\end{array}$ & $\begin{array}{l}\text { Scheimpflug } \\
\text { nuclear density }\end{array}$ & $\begin{array}{l}\text { Visual acuity } \\
\text { (No of letters) }\end{array}$ & $\begin{array}{l}\text { Date } \\
\text { Visit } 2\end{array}$ & Eye & $\begin{array}{l}\text { LOCS II } \\
N O\end{array}$ & $\begin{array}{l}\text { Scheimpflug } \\
\text { nuclear density }\end{array}$ & $\begin{array}{l}\text { Visual acuity } \\
\text { (No of letters) }\end{array}$ \\
\hline 1 & $72 \cdot 1$ & $M$ & $4 / 8 / 91$ & $\mathbf{L}$ & 3 & 0.22 & 59 & $4 / 13 / 92$ & L & 3 & $0 \cdot 42^{\star}$ & 55 \\
\hline 2 & $80 \cdot 1$ & $\mathbf{M}$ & $9 / 16 / 91$ & L & 2 & $0 \cdot 15$ & 55 & $8 / 17 / 92$ & $\overline{\mathrm{L}}$ & 2 & 0.28 & 51 \\
\hline 4 & $59 \cdot 8$ & F & $7 / 1 / 91$ & L & 2 & $0 \cdot 14$ & 50 & $7 / 13 / 92$ & $\mathrm{~L}$ & 2 & 0.22 & 48 \\
\hline 5 & $71 \cdot 8$ & M & $2 / 4 / 91$ & $\mathrm{~L}$ & 1 & 0.08 & 62 & $2 / 24 / 92$ & $\overline{\mathrm{L}}$ & 1 & $0 \cdot 15^{\star}$ & 60 \\
\hline 6 & $48 \cdot 3$ & $\mathbf{F}$ & $7 / 2 / 90$ & L & 3 & 0.30 & 45 & $8 / 5 / 91$ & L & 3 & $0.56^{\star}$ & 33 \\
\hline 7 & $76 \cdot 8$ & $\mathbf{F}$ & $10 / 15 / 90$ & L & 1 & 0.07 & 41 & $10 / 7 / 91$ & L & 1 & 0.07 & 28 \\
\hline 8 & $60 \cdot 6$ & $\mathbf{F}$ & $11 / 19 / 90$ & L & 1 & 0.08 & 58 & $11 / 4 / 91$ & $\bar{L}$ & 1 & 0.09 & 54 \\
\hline 10 & $74 \cdot 3$ & $\mathbf{M}$ & $5 / 6 / 91$ & $\overline{\mathbf{R}}$ & 1 & $0 \cdot 13$ & 55 & $5 / 18 / 92$ & $\mathbf{R}$ & 2 & 0.14 & 56 \\
\hline 11 & $80 \cdot 9$ & $\mathbf{F}$ & $2 / 11 / 91$ & L & 1 & $0 \cdot 12$ & 53 & $2 / 10 / 92$ & $\mathrm{~L}$ & 2 & $0 \cdot 14$ & 49 \\
\hline 12 & $74 \cdot 4$ & $\mathbf{F}$ & $6 / 18 / 90$ & L & 3 & $0 \cdot 20$ & 38 & $5 / 20 / 91$ & L & 2 & $0 \cdot 26^{\star}$ & 38 \\
\hline 13 & $60 \cdot 2$ & F & 9/9/91 & $\mathbf{R}$ & 2 & $0 \cdot 20$ & 54 & $9 / 23 / 92$ & $\mathbf{R}$ & 3 & $0 \cdot 27^{\star}$ & 55 \\
\hline 14 & $58 \cdot 4$ & $\mathbf{M}$ & $6 / 10 / 91$ & $\mathbf{R}$ & 2 & $0 \cdot 26$ & 56 & $6 / 22 / 92$ & $\mathbf{R}$ & 2 & $0 \cdot 40^{\star}$ & 48 \\
\hline 15 & $58 \cdot 8$ & $\mathbf{M}$ & $3 / 11 / 91$ & $\mathbf{R}$ & 3 & $0 \cdot 15$ & 38 & $5 / 11 / 92$ & $\mathbf{R}$ & 2 & $0 \cdot 20^{\star}$ & 33 \\
\hline 16 & 53.4 & M & $10 / 7 / 91$ & $\mathbf{R}$ & 1 & 0.04 & 65 & $9 / 30 / 92$ & $\mathbf{R}$ & 1 & 0.02 & 59 \\
\hline 17 & $67 \cdot 2$ & $\mathbf{M}$ & 9/4/91 & $\mathrm{L}$ & 1 & $0 \cdot 10$ & 53 & $7 / 1 / 92$ & $\mathrm{~L}$ & 1 & $0 \cdot 13^{\star}$ & 49 \\
\hline 18 & $79 \cdot 6$ & F & $8 / 19 / 91$ & $\mathbf{R}$ & 2 & $0 \cdot 16$ & 55 & $9 / 14 / 92$ & $\overline{\mathbf{R}}$ & 1 & $0 \cdot 16$ & 47 \\
\hline 19 & $61 \cdot 7$ & $\mathbf{M}$ & $8 / 19 / 91$ & $\mathbf{L}$ & 2 & 0.33 & 39 & $8 / 10 / 92$ & $\mathrm{~L}$ & 2 & $0.69^{\star}$ & 42 \\
\hline 20 & $50 \cdot 0$ & $\mathrm{~F}$ & $4 / 3 / 91$ & $\overline{\mathbf{L}}$ & 1 & 0.07 & 50 & $5 / 11 / 92$ & $\overline{\mathrm{L}}$ & 1 & 0.09 & 45 \\
\hline 23 & $81 \cdot 4$ & $\mathbf{M}$ & $10 / 7 / 91$ & L & 1 & 0.08 & 38 & $9 / 9 / 92$ & $\overrightarrow{\mathrm{L}}$ & 1 & 0.08 & 35 \\
\hline 24 & $68 \cdot 8$ & $\mathrm{~F}$ & $5 / 6 / 91$ & L & 1 & 0.07 & 35 & $5 / 18 / 92$ & $\mathbf{L}$ & 1 & 0.05 & 38 \\
\hline 25 & $66 \cdot 3$ & $\mathbf{M}$ & $6 / 21 / 91$ & $\mathbf{R}$ & 1 & $0 \cdot 10$ & 55 & $6 / 10 / 92$ & $\overrightarrow{\mathbf{R}}$ & 1 & $0 \cdot 14^{\star}$ & 51 \\
\hline
\end{tabular}

*Significant increase in density. Nuclear cataract data as in Table 1. 
Table 3 Tabulation of data on LOCS II clinical grading of nuclear cataracts which had a one step change on second visit

\begin{tabular}{llll}
\hline & \multicolumn{3}{l}{$\begin{array}{l}\text { LOCS II clinical grading of nuclear } \\
\text { opalescence (visit No) }\end{array}$} \\
\cline { 2 - 4 } $\begin{array}{l}\text { Cataract } \\
\text { case no }\end{array}$ & 1 & 2 & 3 \\
\hline 3 & 1 & 2 & 1 \\
10 & 1 & 2 & $2^{\star}$ \\
11 & 1 & 2 & $2^{\star}$ \\
12 & 3 & 2 & 2 \\
13 & 2 & 2 & 3 \\
15 & 3 & 1 & 2 \\
18 & 2 & 1 & $1^{\star}$ \\
$24 \dagger$ & 0 & 1 & $1^{\star}$ \\
$30 \dagger$ & 0 &
\end{tabular}

^Confirmed LOCS II progression. †Normal control patient. The third visit was required to confirm the visit 2 change using the LOCS II grading. ${ }^{19}$ Visit 1 is baseline, visit 2 is 12 month later, and visit 3 is $18-24$ months from baseline. Only two of the 25 confirmed a 1 step progression.

density, denoting that they progressed at 1 year, and 11 did not show a significant change. None of the 25 regressed. In the normal control group, three of the 30 showed significant increases in density and the rest did not show significant change (Fig 2, Table 1).

With the LOCS II clinical grading, four of 25 cataract cases progressed by one step in nuclear opalescence at 1 year, of which only

Figure 3 Examples of the LOCS II photographs using the Zeiss slit-lamp camera according to the protocol published by Chylack et a ${ }^{18}(A)$ normal control eye No 6 at baseline, (B) at 12 months. LOCS II grading for nuclear opalescence was zero at baseline as well as the first 12 month visit. (C) Nuclear cataract case No 25 at baseline, (B) 12 months. LOCS II grading for nuclear opalescence was 1.0 at baseline and 1.0 at 1 year. (E) Nuclear cataract case No 1 , (F) 12 months. LOCS II grading for nuclear opalescence was 3.0 at baseline and 3.0 after 1 year.

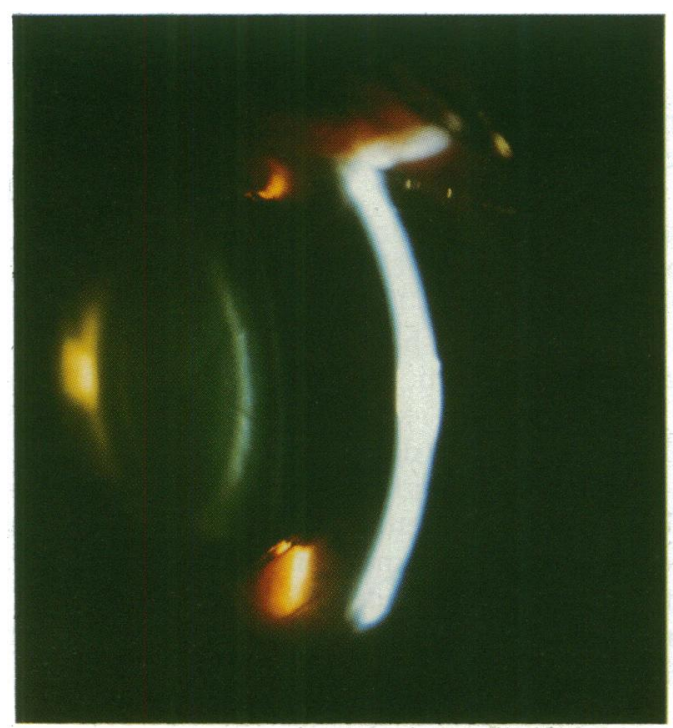

Fig $3 A$

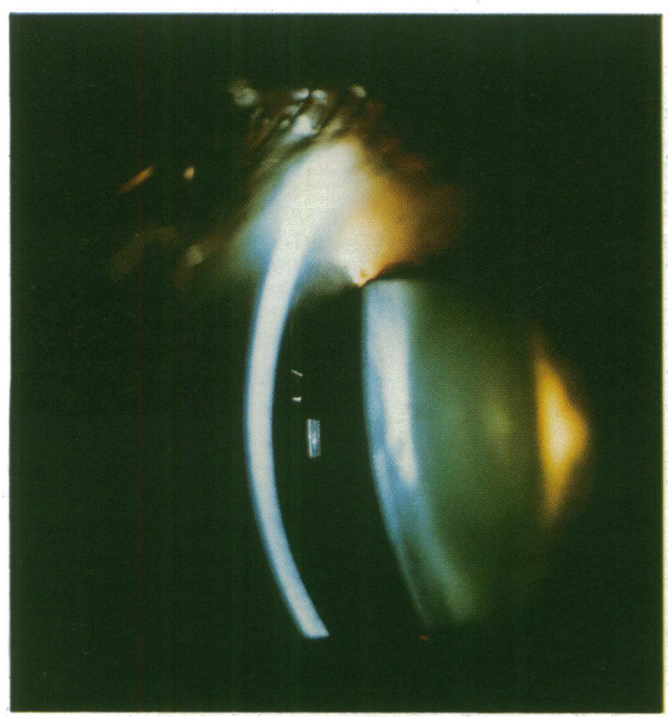

Fig 3C two were confirmed by a third later visit. Two cataract cases showed a regression at 1 year, but were not confirmed by the third visit. These may represent the variability in the system. Three of the normal controls changed in opalescence at 1 year, two of which were confirmed at the third visit. Table 3 shows the data for those eyes which changed showing the results of the third confirmatory visit.

Of the 14 cataractous eyes that progressed by Scheimpflug densitometry, two lost 2 lines ( 5 letters $=1$ line). Of the 11 cataractous eyes which did not progress by Scheimpflug densitometry, one lost 2 lines. Of the three normals that progressed, none lost 2 or more lines of visual acuity and of the 27 normals that did not progress, none lost 2 or more lines of visual acuity. Therefore, three cataractous eyes had clinically significant visual acuity loss (2 lines) compared with none in the control group.

Figures $3 \mathrm{~A}-\mathrm{F}$, are examples of images from three subjects obtained with the Zeiss slit-lamp according to the protocol described by Chylack et $a l,{ }^{18}$ at baseline and at 12 months. Figure $4 \mathrm{~A}-\mathrm{F}$ are corresponding images from the three subjects obtained with the Scheimpflug

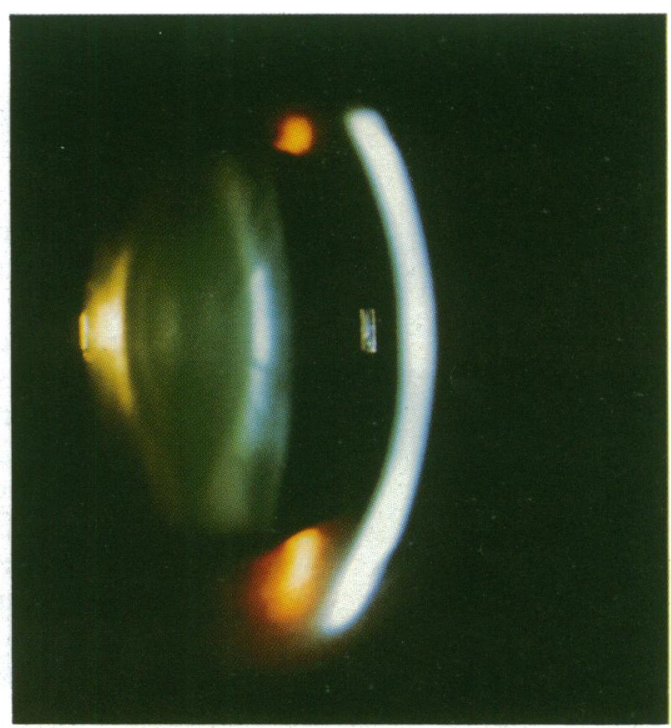

Fig $3 B$

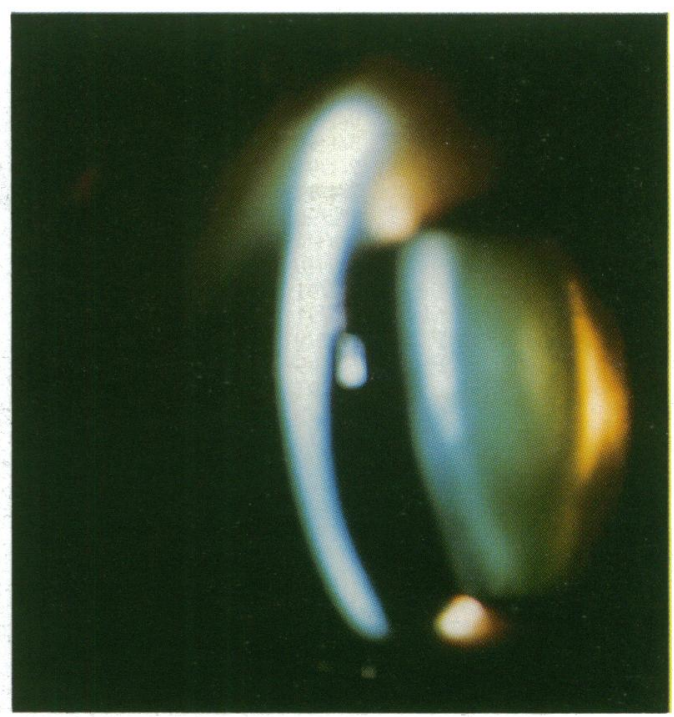

Fig $3 D$ 


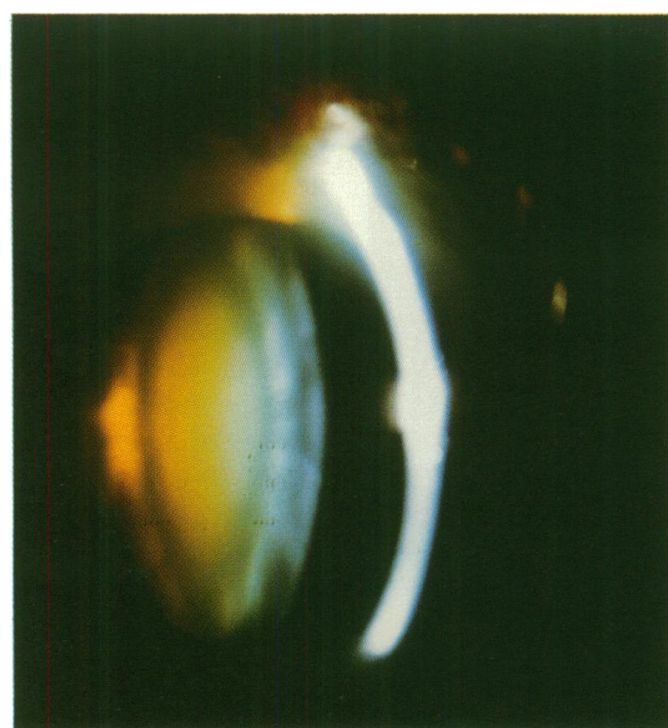

Fig $3 E$

camera. The representative normal control eye ( $3 A, B$ and $4 A, B$ ) shows no progression, whereas the two representative cataractous eyes (3C, D and 4C, D) and (3E, F and $4 E, F$ ) show progression. The normal control eye (3A, B) is from patient no 6 in Table 1 , and the

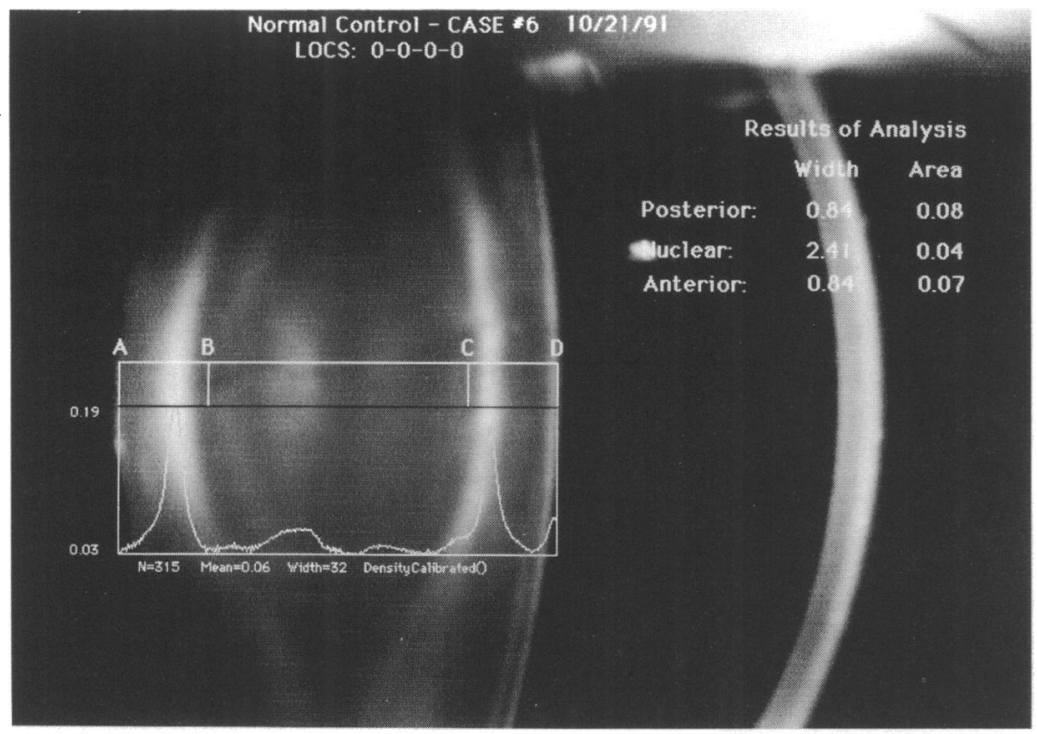

Fig $4 A$

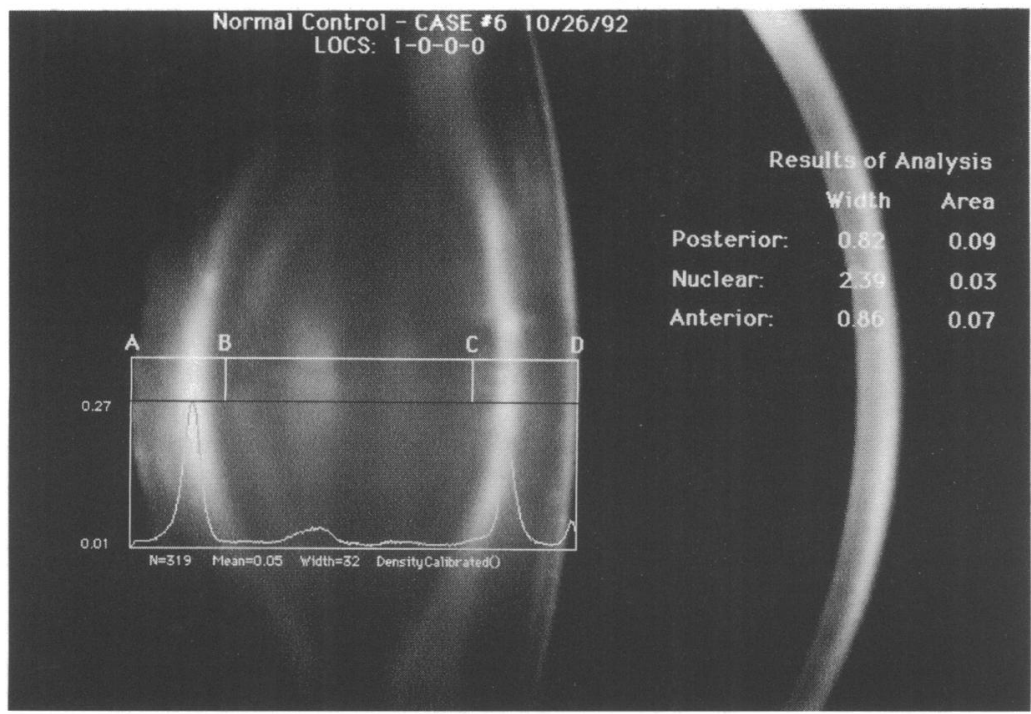

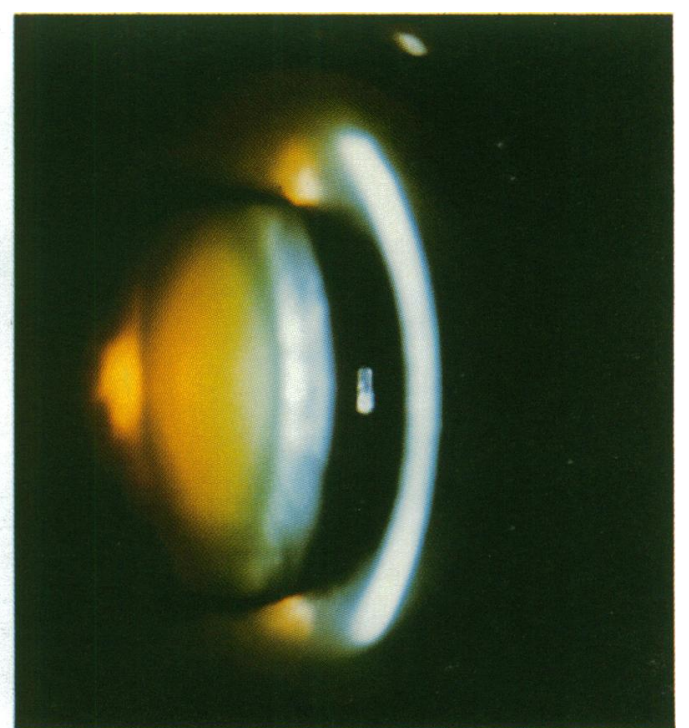

Fig $3 F$

two cataractous eyes (3C, D and $3 E, F)$ are from patient Nos 25 and 1 in Table 2.

\section{Discussion}

These studies suggest that the NEI Scheimpflug system can detect progression of nuclear cataracts within 1 year, changes which may be preclinical and still reversible. This has implications in clinical trials of anticataract drugs since this device can detect the very early changes. For instance, it may show the earliest indication of efficacy of the anticataract drugs being tested before any other clinical variable is affected. It therefore suggests its usefulness as a tool for longitudinal studies such as natural history and interventional studies. In comparison with the LOCS II data, the Scheimpflug method detected significant changes in a larger number of cases compared with LOCS II clinical grading or visual acuity measurements. Among the reasons for this sensitivity may be that the Scheimpflug densitometry uses a continuous scale (in optical density units), and that computerised objective systems like the Scheimpflug system are programmed to pick up smaller density changes which the human eye cannot differentiate. In addition, automated systems like the Scheimpflug system may be more consistent and reliable over time when compared with subjective systems like the LOCS II, and visual acuity testing, which may be prone to problems such as examiner and patient drift, tiredness,

Figure 4 Corresponding Scheimpflug images obtained with the NEI Scheimpflug image analysis system ${ }^{16} 17$ of the eyes shown in Figure 3. Scheimpflug images of normal control No 6, at (A) baseline and at (B) 12 months.

Scheimpflug images of cataract case No 25, at (C) baseline and at (D) 12 months. Scheimpflug images of cataract case No 1, at (E) baseline and (F) 12 months. Note that each image includes the LOCS II grade which is located on the upper left quadrant. The series of numbers (ex: 2-3-0-0) corresponds to nuclear colour (grade 2), nuclear opalescence (grade 3), cortical (grade 0), and post subcapsular (grade 0). The set of numbers on the right upper quadrant correspond to the mean width (in $\mathrm{mm}$ ) and density (in ODU) of the anterior cortex, nucleus, and posterior cortex. For this report, we used only the mean nuclear density from this set of numbers. 


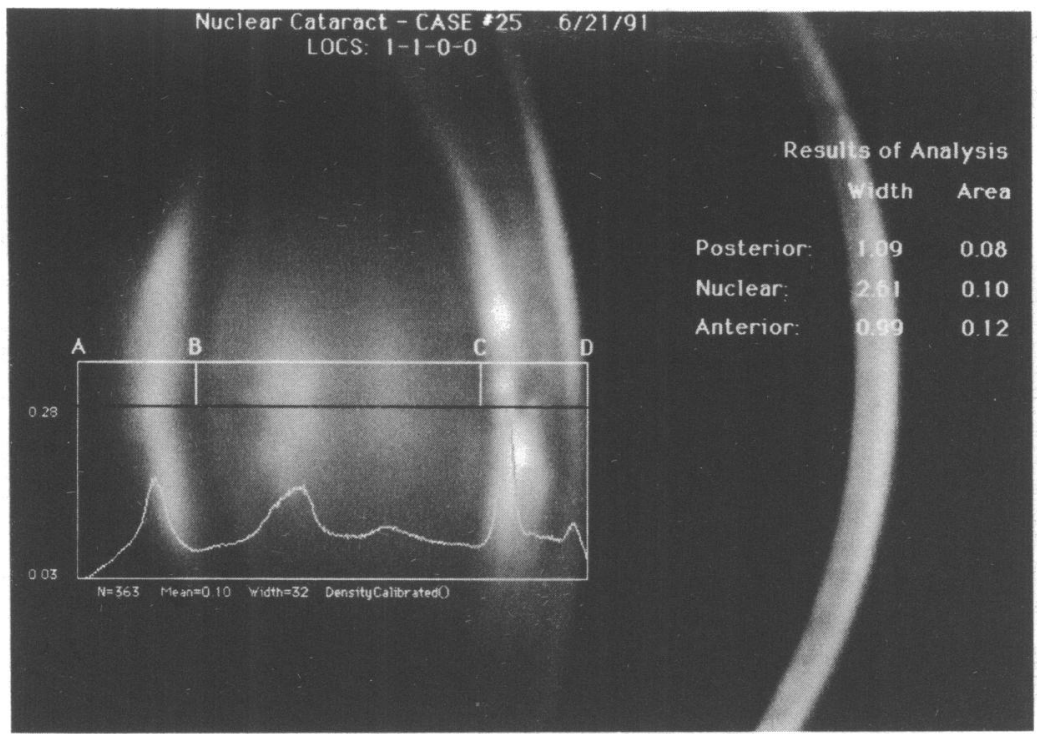

Fig $4 C$

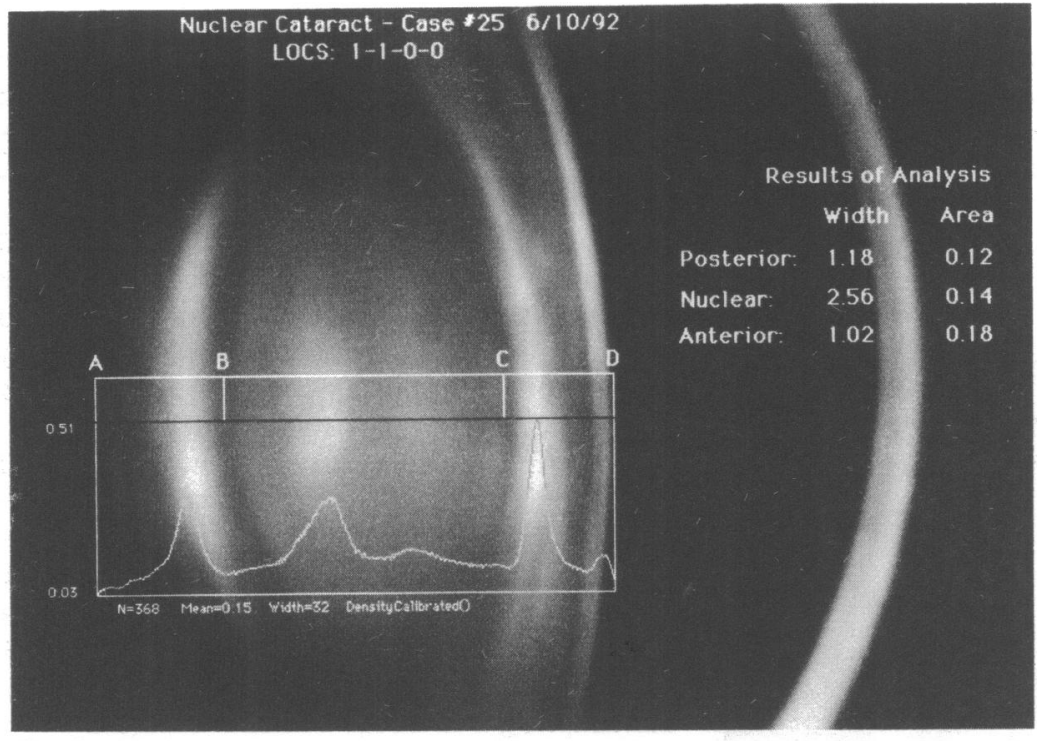

Fig $4 D$

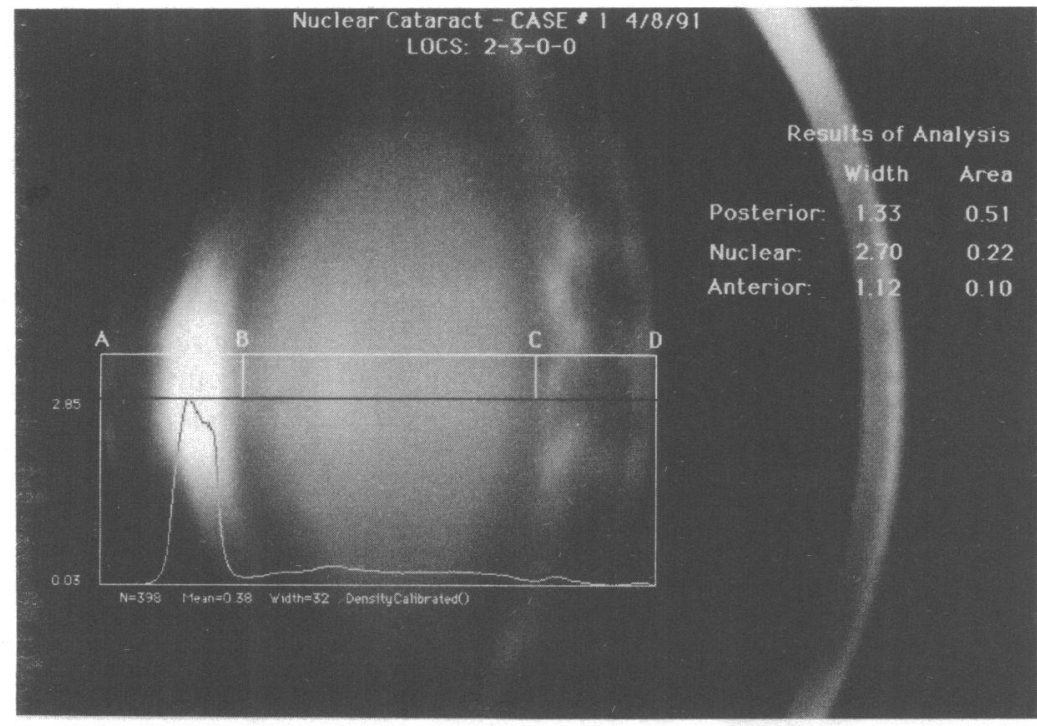

Fig $4 E$

lack of attention, and/or bias. Lastly, the optical disk storage and digital format used in the Scheimpflug system is an important factor contributing to its reproducibility, and has the advantage of dependable, stable, and easily accessible data.
We have previously reported on the studies to convert and express the mean nuclear densities obtained from Scheimpflug photography into optical density units for standardisation, and on the development of a computerised image analysis system (the NEI Scheimpflug cataract imaging system) which separates and delineates the nuclear region apart from the anterior and posterior cortical regions. Conversion of pixel values to optical density units was accomplished by taking images of a series of calibrated neutral density filters $(0.09$ to $1.33 \mathrm{ODU})$ with the existing hardware provided by Zeiss using 13 different illumination levels (from 8 to $14 \mathrm{~V}$ ). The resulting calibration tables then allowed correlation of pixel intensities to optical densities units. ${ }^{1617}$ The details of the software development are described in a previous paper. ${ }^{16}$

Because of the new system's speed, the operator can capture, store, and analyse a cataract image in a few seconds, as well as compare the image analysis with previous image analyses from the same eye. The system, therefore, provides appropriate integrity for the data as well as formats for documenting and monitoring lens opacities. The developments in software were coupled with developments in hardware. We now use a Macintosh Quadra 700 computer and the optical disk storage system.

During the actual usage of the system, it was noted that the upgraded Scheimpflug system had other advantages. With the new Scheimpflug system, the examiner can visualise the image about to be stored and check it for appropriate exposure (using a specially developed exposure check software system) to make sure that the image is within the dynamic range of the camera to allow for future progression, and that the entire lens image is aligned and centred. A poor image can be quickly discarded and the appropriate one obtained. A minor advantage for the patient's viewpoint is that the patient can see his or her cataract at each visit as presented on the computer monitor, and makes their own assessment about the state of their cataract. For many patients, this was very educational and helped them grasp the extent of their eye problem.

The obvious disadvantage of this system is its cost as well as the availability of duplicate equipment for multicentre trials. This may limit its applicability in large scale studies which are usually multicentre. One possible solution to this is the standardisation of a video camera adapted to mount on a slit-lamp and obtain a slit image of the lens which can then be stored and sent to a centre. This system can then perform the analysis using the system we developed. The recently introduced Zeiss video system which can be mounted on any Zeiss slit-lamp is a suitable candidate in this strategy. In theory, the NEI Scheimpflug cataract imaging system may be adopted and modified to analyse any slit image of the lens obtained with other cameras according to certain specifications. This is presently being explored. 


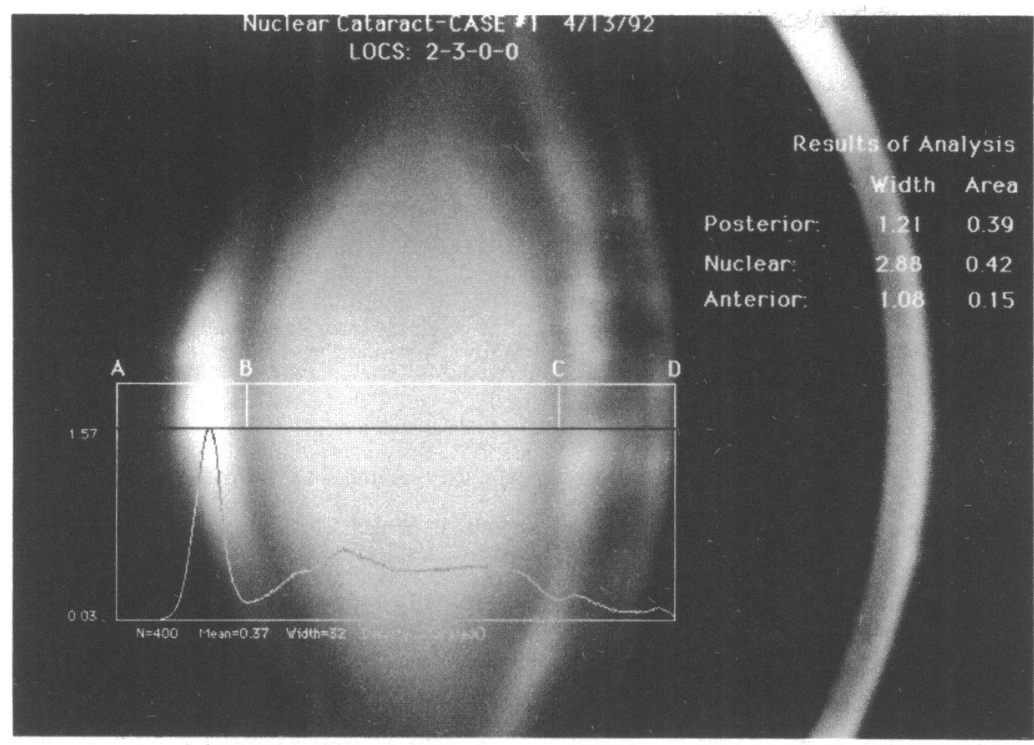

Fig $4 F$

While one of the normal control eyes progressed by the LOCS II clinical grading and by Scheimpflug densitometry, another normal control and two cataract cases progressed by the LOCS II system (Table 3) but did not progress beyond the $99 \%$ range of the Scheimpflug densitometry. One possibility is that perhaps those eyes which progressed only by LOCS II were judged to be exactly equal to the $\mathrm{N} 1$ standard at the baseline visit, and that within a short period of time they progressed slightly over N1 and were graded as N2 in subsequent visits. In the LOCS II system, anything over 0 is graded as 1 , and anything over 1 but not greater than 2 is graded as 2 . Another possible reason may be that the baseline clinical grading may be incorrect, but there is no way to check this. We did perform Scheimpflug densitometry at the third visit $\left(1^{1 / 2}\right.$ to 2 years from baseline), which by then also showed a significant progression. They are as follows: (a) normal control No 24: 0.06 ODU at baseline and $0 \cdot 14$ ODU 2 years later; (b) cataract case No 10: $0 \cdot 13$ ODU at baseline; 0.23 ODU $1^{1 / 2}$ years later; and (c) cataract case number 11: $0 \cdot 12$ ODU at baseline, 0.40 ODU 2 years later.

Recently, Chylack et $a l^{22}$ developed the Lens Opacities Classification System III (LOCS III) for photograding as well as clinical grading $^{24}$ of cataracts, using more standards that are also equally spaced as to increasing severity as an improvement over the LOCS II system. He proposed that it is possibly more sensitive than LOCS II in detecting progression. We are presently conducting studies on this and will report on this in the future. One advantage of photograding is the lesser cost of instrumentation and photographer training, but a skilled, trained, and reliable reader is still needed to grade the photograph. In addition, a system to store, recall, track, and record the photographs ${ }^{10}$ and LOCS grading is needed. These require space, personnel, and additional equipment, but are relatively easy to duplicate.

Although three eyes in the cataract group lost 2 lines of acuity compared with none in the controls, in general, visual acuity was not a sensitive factor in detecting the small lens changes found in this study. These changes are probably subclinical and will need longer follow up periods to allow clinical detection with visual acuity tests. We are presently conducting other studies ${ }^{25-27}$ on cataract patients to correlate type, severity and location of cataracts to changes in visual function, other than Snellen acuity and are following patients for longer periods to determine the amount of change necessary to affect visual acuity significantly.

Recently, Harris et $a l^{28}$ used the Oxford Modular Cataract Image Analysis System, ${ }^{29}$ which has been shown to have excellent reproducibility, ${ }^{30}$ to monitor lens toxicity of the cholesterol lowering agent simvastatin in a clinical trial of the drug taken systemically. They found the Scheimpflug component of the system useful in detecting no untoward effects of the drug in the nuclear portion of the lens within a year, the same period we used in this study. They noted that the 'nuclear dip measure', an index they derived to represent nuclear cataract had $70 \%$ power to detect a change in either direction at a significance level of 0.05 for doubling of the effect.

Although we need to be cautious because of our small numbers in this study, the Scheimpflug imaging method may help identify erstwhile normal patients who may have a higher risk of developing nuclear cataracts. The three normal controls that progressed at 1 year are over 55 years old, and would be more prone to develop cataracts than younger people. This finding could be important in that these three patients may be the ones who will develop definite nuclear cataracts in the near future and would be a good target for anticataract drug treatment. Hence, we plan to pursue this in future studies. One obstacle we encountered was the difficulty of finding normal controls over age 55, since many volunteers were found to have some early lens opacities and therefore were disqualified.

In summary, we found the NEI Scheimpflug cataract imaging system to be useful, sensitive, reproducible, and efficient for monitoring change in the nuclear region of normal and cataractous lenses. However, a longer follow up period will be required to correlate the changes detected by this device to clinically significant change in visual acuity.

The authors have no proprietary interest in any of the instruments or products mentioned in this paper.

We thank Drs Muriel Kaiser-Kupfer and Robert Sperduto for useful discussion, Ms Doretha Leftwood for technical assistance, Mr Mark Vivino and Mr Anup Mahurkar for engineering advice and assistance, Ms Stephanie Jackson and Shirley J Quander for typing the manuscript.

Presented in part at the Association for Research in Vision Presented in part at the Association for Research in
and Ophthalmology Meeting 1993, Sarasota, FL, USA.

1 Datiles MB. Clinical evaluation of cataracts. In: Tasman W, Jaeger EA, eds. Duane's clinical ophthalmology. Philadelphia: JB Lippincott, 1992; 73B: 1-15.

2 Niesel P. Spaltampenphotographic mit der Haagstreit Spaltlampe 900. Ophthalmologica 1966; 151: 489-504.

3 Brown N. Slitlamp photography. Trans Ophthalmol Soc UK 1969; 89: 397-408.

4 Dragomirescu V, Hockwin O, Koch HR, Sasaki K. Development of a new equipment for rotating slit lamp In: Van Hahn, ed. Interdiscip Top Gerontol. Basel: Karger, 1978; 13: 118-30. 
5 Hockwin O, Dragomirescu V, Laser H. Measurement of lens transparency or its disturbances by densitometric analysis of Scheimpflug photographs.

$$
\text { Exp Ophthalmol 1982; 219: 255-62. }
$$

6 Sasaki K, Shibata T. Age related changes of lens transparency: image analysis by photographic sensitometry. In parency: image analysis by photographic sensitometry. In: Henkind P, ed. Proceedings of 14th international congress of ophthalmoll 350 .

7 Sparrow J, Bron AJ, Brown NAP, Ayliffe W, Hill AR. The Oxford clinical cataract classification and grading system. Int Ophthalmol 1986; 9: 207.

8 Brown NAP, Bron AJ, Ayliffe W, Sparrow J, Hill AR. The objective assessment of cataract. Eye 1987; 1: 234-46.

9 Datiles MB, Edwards PA, Trus BL, Green SB. In vivo studies on cataracts using the Scheimpflug slit lamp camera. Invest Ophthalmol Vis Sci 1987; 28: 1707-10.

10 Edwards PA, Datiles MB, Green SB. Reproducibility study on the Scheimpflug cataract video camera. Curr Eye Res 1988; 7: 955-60.

11 Chen SY, Chylack LT, White O. Topcon SL-45 photography: a suitable technique for documenting nuclear, but not cortical cataractous changes in vivo. ARVO Abstracts. Invest Ophthalmol Vis Sci 1985; 26 (suppl): 119.

12 West S, Rosenthal F, Newland HS, Taylor HR. Use of photographic techniques to grade nuclear cataracts. Inves Ophthalmol Vis Sci 1988; 29: 73-7.

13 Edwards PA, Datiles MB, Unser M, Trus BL, Freidlin V, Kashima K. Computerized cataract detection and classification. Curr Eye Res 1990; 9: 517-24.

14 Chylack LT, McCarthy D, Khu P. Use of Topcon SL-45 Scheimpflug slit photography to measure longitudinal growth of nuclear cataracts in vivo. Lens Res 1988; 5: 83-93.

15 Kashima K, Trus BL, Unser M, Edwards PA, Datiles MB Aging studies in the normal lens using the Scheimpflug slit lamp camera. Invest Ophthalmol Vis Sci 1933; 34: 263-9.

16 Vivino MA, Chintalagiri S, Trus BL, Datiles MB Development of a Scheimpflug slit lamp camera system for quantitative densitometric analysis. Eye 1993; 7: 791-8.

17 Magno BV, Freidlin V, Datiles MB. Reproducibility of the
NEI Scheimpflug cataract imaging system. Invest Ophthalmol Vis Sci 1994; 35: 3078-84.

18 Chylack LT, Leske ME, Khu P, Kashiwagi T, Sperduto R. Lens opacities classification system II (LOCS II). Arch Ophthalmol 1989; 107: 991-7.

19 Magno BV, Datiles MB, Lasa SM. Senile cataract progression studies using the Lens Opacities Classificagression studies using the Lens Opacities Classification System

20 Taylor HR, West SK. The clinical grading of lens opacities. Aus NZ $\mathcal{F}$ Ophthalmol 1979; 17: 81-6.

21 Klein BEK, Klein R, Linton KLP, Magli YL, Neider MW. Assessment of cataracts from photography in the Beave Dam Eye Study. Ophthalmology 1990; 97: 1428-33.

22 Chylack LT, Wolfe JK, Singer DM, Leske MC, Bullimore MA, Bailey IL, et al. The Lens Opacities Classification System III. Arch Ophthalmol 1993; 11: 831-6.

23 Ferris R, Kassoff A, Bresnick G, Bailey I. New visual acuity charts for clinical research. Am $\mathcal{F}$ Ophthalmol 1982; 94: charts

24 Karbassi M, Khu P, Singer D, Chylack L. Evaluation of Lens Opacities Classification III applied at the slit lamp. Optom Vis Sci 1993; 70: 923-8.

25 Lasa SM, Datiles MB, Podgor MV, Mango BV, Lee JE. Contrast and glare sensitivity: association with the type and severity of the cataract. Ophthalmology 1992; 99. 1045-9.

26 Drews-Bankiewicz MA, Caruso RD, Datiles MB, KaiserKupfer MI. Contrast sensitivity in patients with nuclear cataracts. Arch Ophthalmol 1992; 110: 953-9.

27 Lasa SM, Podgor MV, Datiles MB, Magno BV. Glare sensitivity in early cataracts. $\mathrm{Br} \mathcal{F}$ Ophthalmol $1993 ; 77$ : 489-91.

28 Harris ML, Brown NAP, Bron AJ. Application of charge couple device Scheimpflug and retroillumination cameras to lens toxicity monitoring in man. Ophthalmic Res 1994; to lens toxicity monitc

29 Sparrow JM, Brown NAP, Shun-Shin GA, Bron AJ. The Oxford modular cataract image analysis system. Eye 1990; 4: $638-48$.

30 Harris ML, Smith GTH, Brown NAP. Inter and intra observer reproducibility of the new Oxford CCD Scheimpflug camera. Eye 1991; 5: 487-90. 